\title{
Anthropometry of Iranian Guidance School Students with Different Ethnicities: A Comparative Study
}

\author{
Amir Houshang Mehrparvar, ${ }^{1}$ Rahmatollah Hafezi, ${ }^{2}$ Seyyed Jalil Mirmohammadi, \\ Mehrdad Mostaghaci, ${ }^{1}$ and Mohammad Hossein Davari ${ }^{1}$
}

${ }^{1}$ Occupational Medicine Department, Shahid Sadoughi University of Medical Sciences, Yazd 8175665563, Iran

${ }^{2}$ Physical Medicine Department, Shahid Beheshti University of Medical Sciences, Tehran, Iran

Correspondence should be addressed to Mehrdad Mostaghaci; mehrdadmostaghaci@gmail.com

Received 1 June 2015; Accepted 30 August 2015

Academic Editor: Malgorzata Wasniewska

Copyright ( 2015 Amir Houshang Mehrparvar et al. This is an open access article distributed under the Creative Commons Attribution License, which permits unrestricted use, distribution, and reproduction in any medium, provided the original work is properly cited.

\begin{abstract}
Objective. We measured some anthropometric dimensions of Iranian guidance school students selected from different ethnicities. Background. Anthropometric dimensions are used for design of equipment, furniture, and clothing. Furniture with inappropriate design not fulfilling the users' anthropometric dimensions may have a negative effect on health. Method. A total of 7400 Iranian guidance school students aged 12-14 years entered the study and their static anthropometric dimensions were measured. Descriptive statistics such as mean, standard deviation, and key percentiles were calculated. All dimensions were compared among different ethnicities and different genders. Results. This study showed significant differences in a set of 22 anthropometric dimensions regarding gender, age, and ethnicity. Conclusion. According to the results of this study, difference between genders and among different ethnicities should be taken into account by designers and manufacturers of guidance school furniture. Application. This study has prepared a data bank of anthropometric dimensions of 12-14-year-old students which can be used as basic information to find appropriate dimensions of school furniture.
\end{abstract}

\section{Introduction}

In order to properly design different products, it is important to have access to anthropometric dimensions of the potential users [1-7].

Inappropriate dimensions of furniture and other products which do not match the users' body dimensions may lead to many adverse effects $[3,5,8-12]$. Students are among the populations at risk for musculoskeletal disorders as a result of incorrect posture, frequently caused by inappropriate school furniture $[6,11]$.

Appropriate design according to the target user groups and identification of the most important body dimensions are key issues in the design process [7]. There is a high prevalence of mismatch between anthropometric data and school furniture which is a factor implicated in causing low back pain [13].

Several researches have worked on anthropometric dimensions of people in different ages in many countries with different races or ethnicities $[9,14-20]$.
It is documented that such factors as age, gender, race, ethnicity, nutrition, and geographic area will affect anthropometric dimensions $[4,21-24]$.

Race refers to grouping of people according to biological characteristics, while ethnicity also encompasses additional cultural factors [25]. Some races consist of different ethnicities with probably different cultural, geographical, economic, and nutritional properties which may eventually create different anthropometric dimensions [5, 24, 26-29]. Anthropometric dimensions in a population may change continuously [14]. Smith and Norris have shown a significant change in body size of the UK children during the past thirty years [30].

There are six ethnicities in different parts of Iran (i.e., Arab, Baluch, Fars, Kurd, Lor, and Turk) with different cultural, economic, nutritional, and geographical characteristics. Turk and Kurd population live in a naturally rich area with cold and damp weather in west and northwest of Iran, and Baluch population live in a deprived area with a hot 

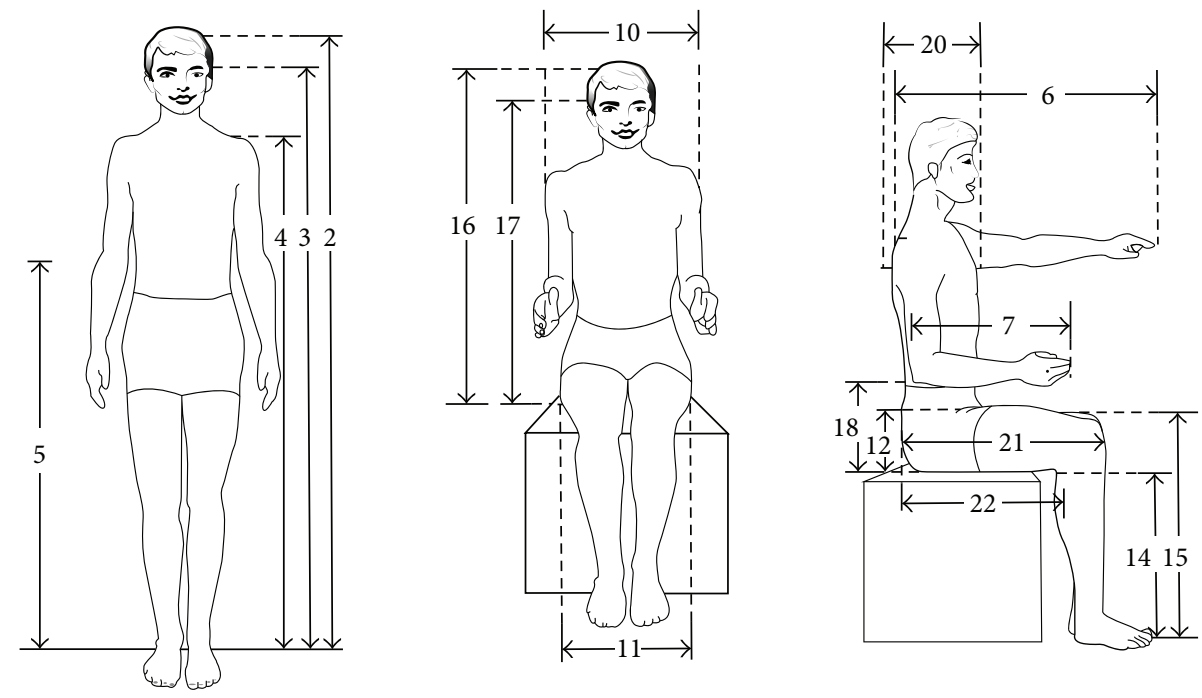

Figure 1

and dry weather in southeast of Iran. It is said that Kurd and Turk population are naturally larger in body size than other ethnicities. The authors have found these differences in their previous study on primary school children in different Iranian ethnicities [24].

Anthropometric studies on Iranian population are few and most of them with small sample size [31,32]. To the best of our knowledge the only large studies for measurement of anthropometric dimensions of students were our previous studies on Iranian primary school children and university students $[24,33]$.

1.1. Purpose. Lack of national anthropometric data leads to the design of clothing, shoes, and furniture based upon anthropometric dimensions of other populations, which may not represent the body sizes of the real population. This study was designed to measure some static anthropometric dimensions in Iranian guidance school children considering ethnic differences.

\section{Material and Methods}

This was a cross-sectional study conducted on 7400 guidance school students aged 12-14 years from different ethnicities in Iran. The students were assigned in each age category according to the information of their identity card; for example, a student was considered to be 12 years old when he (she) was born in the year 1377 Anno Persico (between 21.3.1998 and 20.3.1999 AD).

The dimensions which were measured included the following: weight, standing vertical dimensions (height, eye height, shoulder height, and elbow height), sitting vertical dimensions (popliteal height, knee height, sitting height, eye height, and elbow height), horizontal dimensions (arm

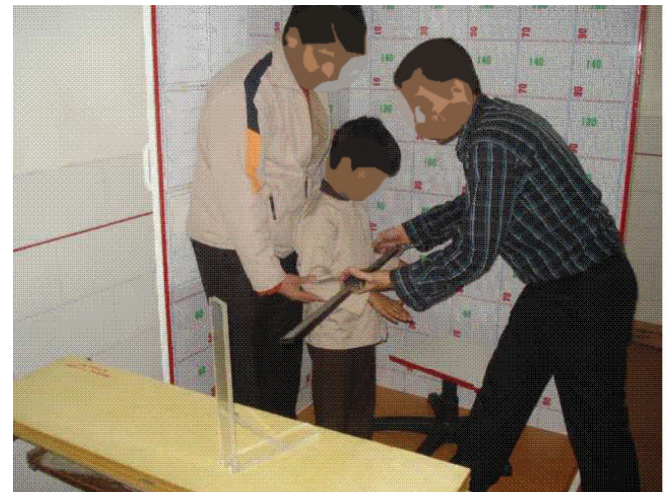

Figure 2

length, forearm length, forearm-forearm distance, elbowelbow distance, shoulder width, buttock width, buttockknee length, and buttock-popliteal length), depths (chest and abdomen), and thicknesses (one-thigh and two-thigh) [24]. Figure 1 shows the measured dimensions.

The subjects stood and sat in standard postures for measurement of standing and sitting dimensions $(27,28)$. Six groups of technicians who were trained for measurements in a planned course performed the measurements using similar techniques. The groups consisted of an observer and two recorders. The dimensions were repeated for $7 \%$ of subjects by two other observers blinded to the previous measurements. Subjects entered the study wearing home clothing without shoes (Figure 2).

2.1. Subjects. The study sample included 7400 subjects $(3560$ boys and 3840 girls) from six ethnicities (1271 Fars, 1234 Kurd, 1342 Lor, 1192 Baluch, 1200 Turk, and 1191 Arab students). The details of the number of subjects are presented in Table 1. Measurements were made during a 4-month period in 2010. 
TABLE 1: Definition of anthropometric data.

\begin{tabular}{|c|c|}
\hline Anthropometric dimensions & Definition \\
\hline (1) Weight & Body weight \\
\hline (2) Body height & Vertical distance from the floor to the vertex (i.e., the crown of the head) \\
\hline (3) Eye height (standing) & Vertical distance from the standing surface to the inner canthus of the eye \\
\hline (4) Shoulder height (standing) & Vertical distance from the standing surface to the shoulder \\
\hline (5) Elbow height (standing) & Vertical distance from the standing surface to the underside of the elbow \\
\hline (6) Arm length & Difference between shoulder height and elbow height \\
\hline (7) Forearm length & Distance between acromion and tip of the middle finger \\
\hline (8) Forearm-forearm distance & Maximum distance between two forearms \\
\hline (9) Elbow-elbow distance & Distance between two acromions in standard sitting position \\
\hline (10) Shoulder width & Maximum shoulder width in standing position \\
\hline (11) Buttock width & Maximum buttock width in sitting position \\
\hline (12) One-thigh thickness & Maximum thickness of the thigh \\
\hline (13) Two-thigh thickness & Maximum two-thigh thickness when right thigh rests over left thigh \\
\hline (14) Popliteal height (sitting) & $\begin{array}{l}\text { Vertical distance from the floor to the popliteal angle at the underside of the knee where the } \\
\text { tendon of the biceps femoris muscle is inserted into the lower leg }\end{array}$ \\
\hline (15) Knee height (sitting) & Vertical distance from the floor to the upper surface of the knee in sitting position \\
\hline (16) Sitting height & Vertical distance from the sitting surface to the vertex \\
\hline (17) Eye height (sitting) & Vertical distance from the sitting surface to the inner canthus of the eye \\
\hline (18) Elbow height (sitting) & Vertical distance from the seat surface to the underside of the elbow \\
\hline (19) Abdominal depth & $\begin{array}{l}\text { Maximum horizontal distance from the vertical reference surface to abdominal front in sitting } \\
\text { position }\end{array}$ \\
\hline (20) Chest depth & $\begin{array}{l}\text { Maximum horizontal distance from the vertical reference plane to the front of the chest in men or } \\
\text { breast in women }\end{array}$ \\
\hline (21) Buttock-knee length & Horizontal distance from the back of the uncompressed buttocks to the front of the kneecap \\
\hline (22) Buttock-popliteal length & $\begin{array}{l}\text { Horizontal distance from the back uncompressed buttocks to the popliteal angle, at the back of } \\
\text { the knee, where the back of the lower legs meets the underside of the thigh }\end{array}$ \\
\hline
\end{tabular}

We obtained an informed written consent from parents and oral consent from students after explanation of the procedure.

2.2. Analysis. Descriptive statistics (mean, standard deviation, and 5, 50, and 95 percentiles) were measured for each dimension regarding gender, age, and ethnicity. The dimensions were compared between two genders and among six ethnicities in each age group. Student's $t$-test and one-way ANOVA were used for the comparison of means between two genders and among six ethnicities, respectively.

\section{Results}

In this study, 7400 subjects ( 3560 boys and 3840 girls) aged 12 to 14 years in six ethnicities were assessed. Table 2 shows means of 22 anthropometric dimensions of guidance school children in 6 different Iranian ethnicities.

Table 3 shows key percentiles (i.e., 5, 50, and 95) for 6 most important anthropometric dimensions (i.e., weight, body height, standing eye height, standing shoulder height, standing elbow height, and buttock-popliteal length) in different ethnicities.
TABLE 2: Sample size of the subjects in different ethnicities and age groups.

\begin{tabular}{lcccccccccc}
\hline & \multicolumn{4}{c}{ Age (year) } & \multicolumn{4}{c}{ Total } \\
Ethnicity & \multicolumn{3}{c}{12} & \multicolumn{2}{c}{13} & \multicolumn{2}{c}{14} & & & \\
& Boys & Girls & Boys & Girls & Boys & Girls & Boys & Girls & Total \\
\hline Fars & 225 & 166 & 223 & 201 & 230 & 246 & 708 & 613 & 1301 \\
Kurd & 152 & 176 & 227 & 204 & 199 & 226 & 578 & 606 & 1184 \\
Lor & 227 & 268 & 227 & 236 & 184 & 200 & 638 & 704 & 1342 \\
Baluch & 126 & 233 & 150 & 252 & 223 & 208 & 499 & 737 & 1192 \\
Turk & 208 & 186 & 203 & 211 & 184 & 208 & 595 & 605 & 1200 \\
Arab & 206 & 132 & 237 & 212 & 129 & 275 & 572 & 619 & 1191 \\
Total & 1144 & 1161 & 1277 & 1316 & 1149 & 1363 & 3560 & 3840 & 7400 \\
\hline
\end{tabular}

There was a significant difference between males and females in most dimensions. Table 4 shows the key percentiles of six more important anthropometric dimensions and Table 5 shows the comparison of dimensions regarding gender.

There was a significant difference between ethnicities in all anthropometric dimensions. $p$ values for difference among ethnicities were less than 0.001 for all dimensions except for 
TABle 3: Mean anthropometric dimensions in Iranian ethnicities.

\begin{tabular}{|c|c|c|c|c|c|c|c|c|c|c|c|c|c|}
\hline \multirow{3}{*}{ Dimensions } & \multirow{3}{*}{ Age } & \multicolumn{12}{|c|}{ Ethnicity } \\
\hline & & \multicolumn{2}{|c|}{ Fars } & \multicolumn{2}{|c|}{ Kurd } & \multicolumn{2}{|c|}{ Lor } & \multicolumn{2}{|c|}{ Baluch } & \multicolumn{2}{|c|}{ Turk } & \multicolumn{2}{|c|}{ Arab } \\
\hline & & Boys & Girls & Boys & Girls & Boys & Girls & Boys & Girls & Boys & Girls & Boys & Girls \\
\hline \multirow{3}{*}{ Weight (kg) } & 12 & 41.82 & 43.10 & 37.49 & 42.51 & 39.08 & 41.62 & 32.13 & 37.83 & 44.26 & 42.12 & 40.67 & 44.89 \\
\hline & 13 & 47.65 & 48.11 & 43.79 & 48.18 & 44.37 & 47.61 & 37.39 & 40.74 & 49.36 & 46.99 & 47.45 & 48.58 \\
\hline & 14 & 54.66 & 52.22 & 49.74 & 50.65 & 52.58 & 51.54 & 41.50 & 44.05 & 57.80 & 49.98 & 51.81 & 52.13 \\
\hline \multirow{3}{*}{ Body height (mm) } & 12 & 1490.86 & 1497.77 & 1456.08 & 1499.94 & 1458.01 & 1490.85 & 1425.42 & 1484.14 & 1512.40 & 1501.73 & 1492.57 & 1507.19 \\
\hline & 13 & 1559.82 & 1534.32 & 1524.44 & 1543.77 & 1536.12 & 1541.77 & 1505.20 & 1516.03 & 1591.87 & 1536.91 & 1563.90 & 1537.40 \\
\hline & 14 & 1644.30 & 1570.52 & 1596.20 & 1572.03 & 1610.86 & 1572.75 & 1546.52 & 1540.60 & 1659.72 & 1573.89 & 1608.75 & 1559.33 \\
\hline \multirow{3}{*}{$\begin{array}{l}\text { Standing eye } \\
\text { height (mm) }\end{array}$} & 12 & & & & & & 1372.79 & 1315.47 & & 1393.55 & & 1359.73 & 1383.48 \\
\hline & 13 & 1444.75 & 1415.34 & 1402.11 & 1422.23 & 1422.51 & 1418.64 & 1392.93 & 1402.20 & 1471.36 & 1416.91 & 1435.21 & 1417.21 \\
\hline & 14 & 1533.58 & 1453.80 & 1472.63 & 1449.84 & 1499.89 & 1452.45 & 1435.80 & 1431.15 & 1543.58 & 1451.46 & 1490.81 & 1435.51 \\
\hline \multirow{3}{*}{$\begin{array}{l}\text { Standing shoulder } \\
\text { height }(\mathrm{mm})\end{array}$} & 12 & 1226.35 & 1242.77 & 1184.40 & 1230.11 & 1198.76 & 1224.29 & 1169.08 & 1217.87 & 1244.18 & 1222.74 & 1216.82 & 1246.66 \\
\hline & 13 & 1286.54 & 1276.84 & 1249.53 & 1271.05 & 1267.62 & 1272.54 & 1241.46 & 1243.80 & 1311.57 & 1242.91 & 1280.25 & 1272.66 \\
\hline & 14 & 1361.47 & 1310.18 & 1313.06 & 1293.96 & 1338.04 & 1300.85 & 1275.40 & 1269.59 & 1376.11 & 1277.86 & 1328.06 & 1292.00 \\
\hline \multirow{3}{*}{$\begin{array}{l}\text { Standing elbow } \\
\text { height (mm) }\end{array}$} & 12 & & & & & & & & & & & & \\
\hline & 13 & 951.90 & 946.31 & 929.16 & 949.55 & 961.54 & 934.32 & 929.19 & 973.69 & 987.82 & 934.33 & 982.45 & 955.97 \\
\hline & 14 & 1016.00 & 968.78 & 975.64 & & 1010.05 & 957.90 & 961.47 & 986.44 & 1035.43 & 962.66 & 1005.96 & 970.58 \\
\hline \multirow{3}{*}{ Chest depth (mm) } & 12 & 175.79 & 182.57 & 174.40 & 197.53 & 163.52 & 178.91 & 161.12 & 175.33 & 186.11 & 200.01 & 173.30 & 201.15 \\
\hline & 13 & 185.68 & 197.94 & 183.12 & 212.41 & 172.73 & 192.97 & 172.33 & 188.36 & 193.55 & 214.96 & 187.98 & 208.59 \\
\hline & 14 & 191.57 & 206.81 & 192.82 & & 184.07 & 197.00 & 174.03 & 197.62 & 203.95 & & 197.87 & 215.22 \\
\hline \multirow{3}{*}{$\begin{array}{l}\text { Abdominal depth } \\
(\mathrm{mm})\end{array}$} & 12 & & & & & & & & & & & & \\
\hline & 13 & & 169.24 & 173.63 & & & 188.78 & 150.42 & 158.81 & 169.62 & & & 175.76 \\
\hline & 14 & & 170.61 & & & & 189.80 & & & & & & 175.67 \\
\hline \multirow{3}{*}{ Arm length (mm) } & 12 & & & 304.09 & & & 307.95 & 298.36 & & 312.76 & 306.25 & 294.38 & 318.20 \\
\hline & 13 & 313.87 & 325.83 & 322.29 & & & 316.47 & 316.03 & 318.21 & 330.14 & 314.11 & 317.24 & 324.43 \\
\hline & 14 & 330.11 & 333.23 & 339.14 & & 329.07 & 322.39 & 327.34 & 326.64 & 348.40 & 319.85 & 323.58 & 329.68 \\
\hline \multirow{3}{*}{$\begin{array}{l}\text { Forearm length } \\
(\mathrm{mm})\end{array}$} & 12 & & & & & & 394.36 & & & 405.22 & & & 402.93 \\
\hline & 13 & & 397.42 & 410.39 & & 404.97 & 409.35 & 406.22 & 391.71 & 428.85 & 409.48 & 417.16 & 413.42 \\
\hline & 14 & 437.87 & 402.45 & 432.13 & & & 412.39 & 419.62 & 405.94 & & & & 416.62 \\
\hline & 12 & 398.77 & 376.28 & 357.38 & 348.48 & 348.94 & 325.97 & 320.41 & 339.58 & 395.67 & 402.30 & 336.00 & 372.69 \\
\hline & 13 & & 388.50 & 376.15 & & & 343.11 & 337.99 & 343.08 & 405.85 & & 357.01 & 389.10 \\
\hline & 14 & 439.14 & 396.52 & 396.49 & & & 335.16 & 347.75 & & & & & 398.10 \\
\hline & 12 & & 315.78 & 370.35 & & 330.13 & 349.27 & 315.69 & 336.93 & 359.99 & & 298.33 & 373.81 \\
\hline & 13 & 385.50 & 328.14 & 392.87 & 373.74 & 343.52 & 370.31 & 330.41 & 341.54 & 372.11 & 373.66 & 323.21 & 388.05 \\
\hline & 14 & 403.61 & 334.42 & 413.43 & 380.86 & 363.91 & 385.85 & 342.07 & 357.91 & 395.26 & 377.19 & 348.88 & 395.76 \\
\hline & 12 & & & 335.59 & & & 335.04 & 321.56 & 339.74 & 345.89 & 318.35 & 328.16 & 351.73 \\
\hline & 13 & 374.75 & 371.54 & 351.41 & 352.25 & 338.28 & 353.13 & 337.21 & 348.54 & 364.68 & 329.78 & 353.24 & 362.76 \\
\hline & 14 & 391.89 & 381.17 & 367.98 & 357.77 & 363.04 & 355.35 & 350.30 & 362.66 & 387.34 & 338.25 & 360.29 & 373.77 \\
\hline & 12 & 271.13 & 287.44 & 288.48 & 306.60 & 272.24 & 281.33 & 257.47 & 270.18 & 293.11 & 311.90 & 270.42 & 303.22 \\
\hline ock width & 13 & 285.79 & 298.86 & 302.34 & 326.50 & 286.74 & 299.75 & 274.60 & 279.53 & 307.81 & 327.75 & 291.82 & 320.23 \\
\hline & 14 & 298.46 & 311.58 & 317.28 & 331.76 & 310.21 & 300.17 & 283.53 & 289.00 & 328.26 & 340.32 & 293.12 & 331.32 \\
\hline & 12 & 112.87 & 98.82 & 105.74 & 118.14 & 99.86 & 78.13 & 93.63 & 116.91 & 108.76 & 111.33 & 97.82 & 112.74 \\
\hline $\begin{array}{l}\mathrm{U} \\
\text { th }\end{array}$ & 13 & 121.78 & 105.33 & 111.60 & 117.27 & 107.86 & 90.30 & 94.31 & 113.33 & 115.70 & 117.60 & 107.52 & 122.76 \\
\hline & 14 & 126.73 & 107.85 & 117.96 & 127.38 & 120.54 & 84.99 & 100.47 & 117.38 & 124.25 & 122.42 & 110.52 & 123.89 \\
\hline & 12 & 235.50 & 205.66 & 197.12 & 225.26 & 187.18 & 188.58 & 181.69 & 198.63 & 220.99 & 199.89 & 216.83 & 195.36 \\
\hline $\mathrm{T}$ & 13 & 237.34 & 215.24 & 207.51 & 240.77 & 196.96 & 204.00 & 181.67 & 196.53 & 226.23 & 209.41 & 240.14 & 203.31 \\
\hline & 14 & 246.63 & 221.75 & 218.27 & 243.07 & 216.95 & 202.79 & 187.47 & 202.75 & 245.96 & 216.77 & 234.86 & 208.19 \\
\hline
\end{tabular}


TABLE 3: Continued.

\begin{tabular}{|c|c|c|c|c|c|c|c|c|c|c|c|c|c|}
\hline \multirow{3}{*}{ Dimensions } & \multirow{3}{*}{ Age } & \multicolumn{12}{|c|}{ Ethnicity } \\
\hline & & \multicolumn{2}{|c|}{ Fars } & \multicolumn{2}{|c|}{ Kurd } & \multicolumn{2}{|c|}{ Lor } & \multicolumn{2}{|c|}{ Baluch } & \multicolumn{2}{|c|}{ Turk } & \multicolumn{2}{|c|}{ Arab } \\
\hline & & Boys & Girls & Boys & Girls & Boys & Girls & Boys & Girls & Boys & Girls & Boys & Girls \\
\hline \multirow{3}{*}{$\begin{array}{l}\text { Popliteal height } \\
(\mathrm{mm})\end{array}$} & 12 & 352.09 & 356.37 & 372.23 & 361.59 & 375.24 & 355.82 & 382.83 & 364.77 & 381.76 & 370.56 & 372.96 & 370.15 \\
\hline & 13 & 362.44 & 361.22 & 391.80 & 365.01 & 400.04 & 368.60 & 399.86 & 367.46 & 400.74 & 373.84 & 387.65 & 381.98 \\
\hline & 14 & 380.76 & 366.19 & 410.10 & 374.44 & 415.21 & 368.53 & 410.14 & 380.50 & 413.07 & 377.69 & 403.25 & 384.58 \\
\hline \multirow{3}{*}{ Knee height (mm) } & 12 & 451.95 & 459.00 & 461.38 & 454.28 & 452.37 & 441.41 & 471.86 & 467.86 & 481.35 & 452.87 & 479.10 & 437.53 \\
\hline & 13 & 471.68 & 467.32 & 486.19 & 458.46 & 480.35 & 458.55 & 480.65 & 473.10 & 502.57 & 458.00 & 504.30 & 449.05 \\
\hline & 14 & 493.72 & 475.90 & 509.29 & 468.12 & 503.09 & 466.16 & 493.60 & 483.86 & 525.35 & 467.28 & 513.52 & 457.85 \\
\hline \multirow{3}{*}{$\begin{array}{l}\text { Buttock-popliteal } \\
\text { length }(\mathrm{mm})\end{array}$} & 12 & 364.23 & 408.00 & 406.28 & 416.28 & 370.61 & 398.52 & 364.81 & 389.31 & 387.45 & 396.56 & 399.81 & 405.98 \\
\hline & 13 & 382.48 & 414.51 & 427.20 & 428.27 & 392.33 & 414.55 & 388.41 & 404.90 & 406.16 & 408.33 & 424.70 & 418.36 \\
\hline & 14 & 407.76 & 425.40 & 446.78 & 437.97 & 415.54 & 422.28 & 403.49 & 409.54 & 426.31 & 421.20 & 431.78 & 429.32 \\
\hline \multirow{3}{*}{$\begin{array}{l}\text { Buttock-knee } \\
\text { length (mm) }\end{array}$} & 12 & 486.69 & 505.09 & 482.40 & 508.31 & 466.82 & 508.94 & 452.87 & 472.63 & 501.40 & 489.27 & 501.77 & 506.70 \\
\hline & 13 & 508.08 & 518.57 & 506.97 & 520.51 & 493.52 & 527.25 & 478.76 & 490.34 & 527.35 & 503.16 & 532.63 & 523.74 \\
\hline & 14 & 533.86 & 533.07 & 529.34 & 531.68 & 523.58 & 535.22 & 493.40 & 500.15 & 552.88 & 516.65 & 537.82 & 531.57 \\
\hline \multirow{3}{*}{$\begin{array}{l}\text { Sitting height } \\
(\mathrm{mm})\end{array}$} & 12 & 764.40 & 785.48 & 769.60 & 777.89 & 771.63 & 766.08 & 711.96 & 757.13 & 769.91 & 787.48 & 750.34 & 797.83 \\
\hline & 13 & 801.90 & 807.71 & 799.11 & 812.21 & 799.69 & 791.35 & 751.96 & 778.63 & 815.73 & 809.08 & 779.43 & 819.52 \\
\hline & 14 & 846.56 & 833.61 & 828.51 & 824.04 & 838.42 & 806.45 & 780.54 & 793.99 & 843.50 & 833.32 & 807.22 & 829.08 \\
\hline \multirow{3}{*}{$\begin{array}{l}\text { Sitting eye height } \\
(\mathrm{mm})\end{array}$} & 12 & 649.02 & 666.29 & 652.16 & 665.79 & 656.78 & 654.02 & 611.73 & 643.48 & 650.04 & 674.43 & 631.37 & 673.75 \\
\hline & 13 & 690.35 & 691.79 & 679.58 & 697.27 & 686.91 & 671.35 & 649.86 & 666.78 & 697.43 & 695.54 & 657.72 & 698.72 \\
\hline & 14 & 738.26 & 716.01 & 709.34 & 705.27 & 725.32 & 678.15 & 674.86 & 681.71 & 726.78 & 719.01 & 688.72 & 708.42 \\
\hline \multirow{3}{*}{$\begin{array}{l}\text { Sitting elbow } \\
\text { height }(\mathrm{mm})\end{array}$} & 12 & 173.00 & 199.24 & 195.98 & 189.32 & 203.30 & 174.70 & 179.40 & 217.07 & 201.40 & 205.59 & 180.52 & 204.00 \\
\hline & 13 & 186.09 & 208.08 & 204.85 & 196.01 & 212.59 & 182.92 & 190.27 & 230.83 & 232.80 & 214.28 & 180.71 & 212.76 \\
\hline & 14 & 200.36 & 222.90 & 211.33 & 200.24 & 221.68 & 185.70 & 205.21 & 238.60 & 231.57 & 231.65 & 186.74 & 220.41 \\
\hline
\end{tabular}

Bold and italic prints show the highest and lowest values, respectively, in each age group and gender.

height in 12-year-old girls ( $p=0.260$ ), sitting height in 12year-old girls $(p=0.519)$, and sitting eye height in 13-yearold girls $(p=0.030)$.

\section{Discussion}

Today, it is important to measure anthropometric dimensions as a key step for design process. Such variables as age, gender, and ethnicity affect these dimensions, so it is critical to consider these variables for preparation of anthropometric databases.

In this study we measured the anthropometric dimensions of students aged 12 to 14 years from different ethnicities in Iran. The results showed a significant difference between two genders in all age groups and among all ethnicities for most of the dimensions.

In this study, 12- and 13-year-old girls' weight was significantly more than boys which was opposite in 14-year-old subjects. In 12-year-old subjects, girls had larger heights and upper extremity lengths, although boys showed larger lower extremity heights and lengths. In 13- and 14-year-old subjects, most anthropometric dimensions were significantly larger in boys except for depths and dimensions related to buttocks which is explainable by the size of breasts and buttocks in girls due to puberty.
In this study, Turk boys had the largest dimensions in most of the measured dimensions except for abdominal depth, upper extremity distances and widths, lower extremity, and sitting heights which were larger in Fars ethnicity, although in some of them such as forearm-forearm distance the difference was negligible. Most dimensions were smaller in Baluch boys than other ethnicities except for most upper extremity dimensions and some lower extremity heights.

In about one-third of dimensions Arab girls had the largest measures and in other dimensions Kurds and Turks showed larger dimensions.

There is a considerable difference among different Iranian ethnicities regarding genetic characteristics, climate, geographical area, and socioeconomic characteristics which may affect anthropometric dimensions. For example, Turks and Kurds live in a naturally rich area, but Baluchis live in a province which is naturally deprived, so some differences are probably due to these issues.

There are some studies in different populations for measurement of anthropometric dimensions. Mokdad and AlAnsari measured 44 anthropometric dimensions of Bahraini children aged 6-12 years. They found significant difference between two genders in many dimensions [34]. A total of 50 anthropometric dimensions were measured among Mexican children and a significant difference among different populations was found [16]. 
TABLE 4: Key percentiles of six anthropometric dimensions.

\begin{tabular}{|c|c|c|c|c|c|c|c|c|c|c|c|}
\hline \multirow{4}{*}{ Dimension } & \multirow{4}{*}{ Ethnicity } & \multirow{4}{*}{ Sex } & \multicolumn{9}{|c|}{ Age } \\
\hline & & & \multirow{2}{*}{\multicolumn{3}{|c|}{$\begin{array}{c}12 \\
\text { Percentile }\end{array}$}} & \multirow{2}{*}{\multicolumn{3}{|c|}{$\begin{array}{c}13 \\
\text { Percentile }\end{array}$}} & \multirow{2}{*}{\multicolumn{3}{|c|}{$\begin{array}{c}14 \\
\text { Percentile }\end{array}$}} \\
\hline & & & & & & & & & & & \\
\hline & & & 5 & 50 & 95 & 5 & 50 & 95 & 5 & 50 & 95 \\
\hline \multirow{12}{*}{ Weight (Kg) } & \multirow{2}{*}{ Fars } & Boys & 28.16 & 39.10 & 61.94 & 31.00 & 45.50 & 68.90 & 36.90 & 52.85 & 80.63 \\
\hline & & Girls & 27.71 & 42.55 & 63.91 & 32.11 & 46.70 & 69.80 & 38.43 & 50.40 & 71.00 \\
\hline & \multirow{2}{*}{ Lor } & Boys & 28.00 & 37.00 & 56.20 & 30.00 & 42.00 & 65.60 & 35.00 & 49.50 & 77.75 \\
\hline & & Girls & 28.00 & 41.00 & 59.55 & 31.85 & 46.00 & 67.15 & 37.05 & 50.00 & 68.90 \\
\hline & \multirow{2}{*}{ Kurd } & Boys & 28.19 & 35.65 & 54.51 & 30.74 & 41.80 & 64.05 & 34.20 & 48.10 & 73.10 \\
\hline & & Girls & 28.58 & 41.40 & 62.36 & 33.00 & 47.05 & 68.80 & 38.73 & 49.00 & 67.29 \\
\hline & \multirow{2}{*}{ Turk } & Boys & 31.09 & 41.80 & 66.05 & 34.42 & 47.70 & 70.00 & 41.17 & 55.65 & 85.45 \\
\hline & & Girls & 29.63 & 40.75 & 58.93 & 32.00 & 46.40 & 65.96 & 36.10 & 48.40 & 68.77 \\
\hline & \multirow{2}{*}{ Baluch } & Boys & 25.00 & 31.00 & 44.30 & 27.05 & 36.00 & 53.00 & 28.04 & 40.00 & 60.80 \\
\hline & & Girls & 26.70 & 36.0 & 52.00 & 28.00 & 40.00 & 56.00 & 31.45 & 43.00 & 59.00 \\
\hline & \multirow{2}{*}{ Arab } & Boys & 28.00 & 38.75 & 58.00 & 32.45 & 46.00 & 68.00 & 35.00 & 51.00 & 76.00 \\
\hline & & Girls & 29.79 & 43.50 & 63.74 & 33.63 & 46.85 & 75.17 & 39.36 & 50.80 & 69.86 \\
\hline \multirow{12}{*}{ Body height (mm) } & \multirow{2}{*}{ Fars } & Boys & 1370.00 & 1490.00 & 1620.00 & 1410.00 & 1560.00 & 1710.00 & 1495.50 & 1645.00 & 1784.50 \\
\hline & & Girls & 1380.25 & 1510.00 & 1595.00 & 1430.00 & 1540.00 & 1634.50 & 1481.75 & 1570.00 & 1655.00 \\
\hline & \multirow{2}{*}{ Lor } & Boys & 1360.00 & 1460.00 & 1560.00 & 1400.00 & 1540.00 & 1680.00 & 1462.50 & 1600.00 & 1750.00 \\
\hline & & Girls & 1360.00 & 1495.00 & 1610.00 & 1420.00 & 1550.00 & 1641.50 & 1480.00 & 1570.00 & 1679.50 \\
\hline & Kurd & Boys & 1360.00 & 1445.00 & 1606.75 & 1395.00 & 1525.00 & 1683.00 & 1445.00 & 1595.00 & 1740.00 \\
\hline & & Girls & 1390.00 & 1500.00 & 1620.75 & 1437.50 & 1540.00 & 1655.00 & 1486.75 & 1570.00 & 1655.00 \\
\hline & & Boys & 1382.25 & 1510.00 & 1662.75 & 1456.00 & 1590.00 & 1734.00 & 1530.00 & 1665.00 & 1780.00 \\
\hline & Ituin & Girls & 1370.00 & 1505.00 & 1613.25 & 1423.00 & 1540.00 & 1640.00 & 1482.25 & 1575.00 & 1672.75 \\
\hline & & Boys & 1300.00 & 1420.00 & 1569.50 & 1360.00 & 1502.50 & 1687.25 & 1390.00 & 1550.00 & 1700.00 \\
\hline & ( & Girls & 1367.00 & 1490.00 & 1610.00 & 1400.00 & 1520.00 & 1640.00 & 1442.25 & 1550.00 & 1630.00 \\
\hline & Arab & Boys & 1370.00 & 1490.00 & 1636.50 & 1420.00 & 1560.00 & 1715.00 & 1442.50 & 1620.00 & 1740.00 \\
\hline & 1100 & Girls & 1374.75 & 1510.00 & 1601.75 & 1425.00 & 1540.00 & 1633.50 & 1450.00 & 1560.00 & 1660.00 \\
\hline & Fars & Boys & 1255.00 & 1370.00 & 1498.50 & 1295.00 & 1450.00 & 1600.00 & 1390.00 & 1530.00 & 1684.50 \\
\hline & 1010 & Girls & 1248.50 & 1385.00 & 1475.00 & 1310.00 & 1420.00 & 1505.00 & 1360.00 & 1450.00 & 1550.00 \\
\hline & & Boys & 1240.00 & 1340.00 & 1450.00 & 1280.00 & 1430.00 & 1570.00 & 1352.50 & 1495.00 & 1640.00 \\
\hline & LOI & Girls & 1240.00 & 1370.00 & 1490.00 & 1300.00 & 1420.00 & 1521.50 & 1370.5 & 1450.00 & 1560.00 \\
\hline & & Boys & 1240.00 & 1330.00 & 1473.50 & 1272.00 & 1400.00 & 1552.00 & 1335.00 & 1475.00 & 1615.00 \\
\hline Standing e & & Girls & 1264.25 & 1375.00 & 1500.00 & 1316.25 & 1420.00 & 1542.50 & 1353.50 & 1450.00 & 1540.00 \\
\hline tanining 4 & Turk & Boys & 1255.00 & 1390.00 & 1538.25 & 1330.00 & 1480.00 & 1623.00 & 1411.25 & 1550.00 & 1673.75 \\
\hline & 14ne & Girls & 1253.50 & 1390.00 & 1485.00 & 1310.00 & 1425.00 & 1520.00 & 1352.25 & 1450.00 & 1555.00 \\
\hline & & Boys & 1186.75 & 1310.00 & 1466.00 & 1245.50 & 1382.50 & 1574.50 & 1270.00 & 1440.00 & 1600.00 \\
\hline & Darucin & Girls & 1250.00 & 1370.00 & 1513.00 & 1266.50 & 1405.00 & 1540.00 & 1332.25 & 1437.50 & 1525.50 \\
\hline & 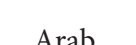 & Boys & 1230.00 & 1350.00 & 1530.00 & 1289.00 & 1430.00 & 1600.00 & 1305.00 & 1505.00 & 1635.00 \\
\hline & Milav & Girls & 1240.00 & 1390.00 & 1481.75 & 1300.00 & 1420.00 & 1520.00 & 1325.00 & 1435.00 & 1535.00 \\
\hline & & Boys & 1110.00 & 1220.00 & 1345.00 & 1152.00 & 1290.00 & 1414.00 & 1235.50 & 1360.00 & 1480.00 \\
\hline & & Girls & 1125.00 & 1250.00 & 1340.00 & 1170.50 & 1285.00 & 1365.00 & 1231.75 & 1310.00 & 1395.00 \\
\hline & Lor & Boys & 1104.00 & 1200.00 & 1296.00 & 1120.00 & 1270.00 & 1400.00 & 1200.00 & 1340.00 & 1467.50 \\
\hline & & Girls & 1094.50 & 1230.00 & 1330.00 & 1170.00 & 1280.00 & 1370.00 & 1220.00 & 1300.00 & 1399.50 \\
\hline & & Boys & 1095.00 & 1175.00 & 1310.50 & 1132.00 & 1250.00 & 1394.00 & 1180.00 & 1310.00 & 1455.00 \\
\hline & & Girls & 1119.25 & 1230.00 & 1340.75 & 1171.25 & 1260.00 & 1377.50 & 1213.50 & 1295.00 & 1383.25 \\
\hline 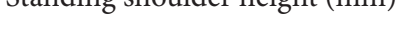 & Turk & Boys & 1135.00 & 1245.00 & 1380.50 & 1190.00 & 1310.00 & 1440.00 & 1240.00 & 1380.00 & 1495.00 \\
\hline & 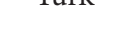 & Girls & 1106.75 & 1222.50 & 1323.25 & 1140.00 & 1240.00 & 1340.00 & 1190.00 & 1275.00 & 1380.00 \\
\hline & Baluch & Boys & 1050.00 & 1170.00 & 1306.50 & 1105.50 & 1240.00 & 1410.00 & 1120.00 & 1290.00 & 1428.00 \\
\hline & & Girls & 1110.00 & 1220.00 & 1336.50 & 1126.50 & 1250.00 & 1353.50 & 1184.50 & 1270.00 & 1350.00 \\
\hline & Arab & Boys & 1110.00 & 1210.00 & 1340.00 & 1150.00 & 1280.00 & 1420.00 & 1180.00 & 1340.00 & 1440.00 \\
\hline & & Girls & 1123.00 & 1250.00 & 1333.50 & 1175.00 & 1272.50 & 1360.00 & 1199.00 & 1300.00 & 1380.00 \\
\hline
\end{tabular}


TABLE 4: Continued.

\begin{tabular}{|c|c|c|c|c|c|c|c|c|c|c|c|}
\hline \multirow{4}{*}{ Dimension } & \multirow{4}{*}{ Ethnicity } & \multirow{4}{*}{ Sex } & \multicolumn{9}{|c|}{ Age } \\
\hline & & & \multirow{2}{*}{\multicolumn{3}{|c|}{$\begin{array}{c}12 \\
\text { Percentile }\end{array}$}} & \multirow{2}{*}{\multicolumn{3}{|c|}{$\begin{array}{c}13 \\
\text { Percentile }\end{array}$}} & \multirow{2}{*}{\multicolumn{3}{|c|}{$\begin{array}{c}14 \\
\text { Percentile }\end{array}$}} \\
\hline & & & & & & & & & & & \\
\hline & & & 5 & 50 & 95 & 5 & 50 & 95 & 5 & 50 & 95 \\
\hline \multirow{12}{*}{ Standing elbow height (mm) } & \multirow{2}{*}{ Fars } & Boys & 820.00 & 910.00 & 1000.00 & 840.00 & 950.00 & 1040.00 & 911.00 & 1020.00 & 1110.00 \\
\hline & & Girls & 836.50 & 925.00 & 1005.00 & 870.25 & 950.00 & 1020.00 & 900.00 & 970.00 & 1035.00 \\
\hline & \multirow{2}{*}{ Lor } & Boys & 830.00 & 910.00 & 1000.00 & 860.00 & 960.00 & 1080.00 & 910.00 & 1010.00 & 1100.00 \\
\hline & & Girls & 804.50 & 900.00 & 990.00 & 840.00 & 940.00 & 1020.00 & 900.00 & 950.00 & 1030.00 \\
\hline & \multirow{2}{*}{ Kurd } & Boys & 808.25 & 875.00 & 973.50 & 840.00 & 925.00 & 1025.00 & 880.00 & 980.00 & 1070.00 \\
\hline & & Girls & 840.00 & 920.00 & 1006.00 & 880.00 & 945.00 & 1034.00 & 900.00 & 955.00 & 1020.00 \\
\hline & \multirow{2}{*}{ Turk } & Boys & 840.00 & 935.00 & 1040.00 & 901.00 & 990.00 & 1088.00 & 877.50 & 1020.00 & 1100.00 \\
\hline & & Girls & 830.00 & 920.00 & 1000.00 & 843.00 & 940.00 & 1012.00 & 902.25 & 960.00 & 1040.00 \\
\hline & \multirow{2}{*}{ Baluch } & Boys & 783.50 & 880.00 & 976.50 & 832.75 & 930.00 & 1054.50 & 840.00 & 970.00 & 1088.0 \\
\hline & & Girls & 867.00 & 950.00 & 1050.00 & 896.50 & 980.00 & 1070.00 & 904.50 & 990.00 & 1080.00 \\
\hline & \multirow{2}{*}{ Arab } & Boys & 820.00 & 925.00 & 1056.50 & 880.00 & 980.00 & 1090.00 & 920.00 & 1040.00 & 1130.00 \\
\hline & & Girls & 780.00 & 940.00 & 1000.00 & 890.00 & 955.00 & 1031.75 & 900.00 & 970.00 & 1050.00 \\
\hline \multirow{12}{*}{ Buttock-popliteal length (mm) } & \multirow{2}{*}{ Fars } & Boys & 320.00 & 362.00 & 418.00 & 330.20 & 380.00 & 434.00 & 350.55 & 408.00 & 464.90 \\
\hline & & Girls & 355.40 & 411.00 & 458.30 & 365.40 & 415.00 & 465.00 & 379.35 & 424.00 & 471.00 \\
\hline & \multirow{2}{*}{ Lor } & Boys & 330.00 & 370.00 & 420.00 & 344.00 & 390.00 & 450.00 & 370.00 & 410.00 & 477.50 \\
\hline & & Girls & 443.00 & 512.50 & 568.55 & 358.80 & 412.00 & 476.60 & 375.00 & 420.00 & 472.85 \\
\hline & \multirow{2}{*}{ Kurd } & Boys & 369.00 & 404.50 & 449.70 & 373.80 & 428.00 & 480.60 & 396.00 & 446.00 & 499.00 \\
\hline & & Girls & 357.80 & 418.50 & 474.90 & 369.75 & 427.50 & 484.00 & 380.00 & 438.00 & 488.00 \\
\hline & \multirow{2}{*}{ Turk } & Boys & 342.00 & 387.00 & 441.20 & 350.20 & 408.00 & 450.00 & 383.00 & 425.50 & 473.25 \\
\hline & & Girls & 359.00 & 394.50 & 432.00 & 369.10 & 409.50 & 452.00 & 386.00 & 422.00 & 458.00 \\
\hline & \multirow{2}{*}{ Baluch } & Boys & 319.70 & 363.50 & 421.25 & 327.75 & 387.00 & 448.45 & 348.00 & 402.50 & 473.00 \\
\hline & & Girls & 326.20 & 392.00 & 437.90 & 343.90 & 407.00 & 459.00 & 353.00 & 410.00 & 460.55 \\
\hline & \multirow{2}{*}{ Arab } & Boys & 326.75 & 400.00 & 470.00 & 369.50 & 420.00 & 490.00 & 362.50 & 430.00 & 490.00 \\
\hline & & Girls & 338.38 & 406.00 & 456.70 & 363.24 & 418.55 & 468.36 & 387.26 & 428.50 & 476.84 \\
\hline
\end{tabular}

The difference between races or ethnicities regarding anthropometric dimensions has also been identified in some studies. Rosnah et al. found a significant difference between Malays and non-Malays which is in agreement with the results of current study [35]. Jahanshahi et al. found that ethnicity affects facial anthropometric dimensions in an Iranian population [27]. Lin et al. also found a significant difference among four East Asian populations [5].

Although climatic, nutritional, and economic factors are significantly different in different populations and countries, one of the important factors contributing to anthropometric differences is race or ethnicity.

Anthropometric dimensions of the study children were different from other populations. Anthropometric dimensions of Greek, American, and Mexican children were more than Iranian children $[16,17,19]$ and these dimensions in Vietnamese children were less than Iranian children [9].

The anthropometric dimensions we measured in this study can be used to design school furniture matched to our population. It is recommended that the number of anthropometric dimensions be increased to create databases used to design clothing, shoes, and other products. It is also recommended that these measurements be repeated to seek for temporal trends.
This study had some limitations. We tried to select real native students in each ethnicity, but some hybrid students may have entered the study and we might not have detected these subjects.

\section{Conclusions}

In this study we found significantly different anthropometric dimensions in different Iranian ethnicities. This makes it necessary to pay attention to these differences when school furniture is designed. The results of this study showed that students need furniture specifically designed and manufactured considering their ethnicity, gender, and age. It is obvious that designing school furniture separately for each ethnicity is very difficult, so a practical approach is to design adjustable furniture using the anthropometric dimension measured in this study.

\section{Key Points}

(i) Anthropometric dimensions of the users should be put in mind when determining the dimensions of school furniture. 
TABLE 5: Comparison of anthropometric dimensions between two genders.

\begin{tabular}{|c|c|c|c|c|c|c|c|c|c|}
\hline \multirow{3}{*}{ Dimensions } & \multicolumn{9}{|c|}{ Age (year) } \\
\hline & \multicolumn{3}{|c|}{12} & \multicolumn{3}{|c|}{13} & \multicolumn{3}{|c|}{14} \\
\hline & Boys & Girls & $p$ & Boys & Girls & $p$ & Boys & Girls & $p$ \\
\hline Weight (kg) & 39.87 & 41.66 & $<0.001$ & 45.39 & 46.52 & 0.012 & 51.10 & 50.25 & 0.072 \\
\hline Body height (mm) & 1476.74 & 1495.47 & $<0.001$ & 1548.67 & 1534.53 & $<0.001$ & 1610.12 & 1564.79 & $<0.001$ \\
\hline Standing eye height $(\mathrm{mm})$ & 1357.33 & 1377.30 & $<0.001$ & 1429.47 & 1415.04 & $<0.001$ & 1595.45 & 1445.44 & $<0.001$ \\
\hline Standing shoulder height (mm) & 1210.52 & 1228.82 & $<0.001$ & 1274.02 & 1262.73 & $<0.001$ & 1331.22 & 1291.32 & $<0.001$ \\
\hline Standing elbow height (mm) & 912.94 & 922.81 & $<0.001$ & 958.31 & 949.54 & $<0.001$ & 999.61 & 967.63 & $<0.001$ \\
\hline Chest depth (mm) & 172.98 & 187.45 & $<0.001$ & 183.02 & 201.90 & $<0.001$ & 188.74 & 210.58 & $<0.001$ \\
\hline Abdominal depth (mm) & 160.97 & 168.87 & $<0.001$ & 168.62 & 174.09 & $<0.001$ & 173.51 & 175.92 & 0.058 \\
\hline Arm length $(\mathrm{mm})$ & 300.22 & 311.69 & $<0.001$ & 318.65 & 320.43 & 0.071 & 333.17 & 327.26 & $<0.001$ \\
\hline Forearm length (mm) & 388.62 & 393.23 & $<0.001$ & 413.74 & 404.92 & $<0.001$ & 431.91 & 411.77 & $<0.001$ \\
\hline Forearm-forearm distance $(\mathrm{mm})$ & 362.91 & 356.85 & 0.003 & 376.65 & 372.02 & 0.017 & 395.98 & 379.94 & $<0.001$ \\
\hline Elbow-elbow distance (mm) & 341.33 & 348.07 & $<0.001$ & 358.98 & 362.27 & 0.090 & 375.04 & 369.22 & 0.004 \\
\hline Shoulder width (mm) & 334.52 & 339.01 & 0.001 & 353.95 & 352.74 & 0.364 & 370.78 & 362.64 & $<0.001$ \\
\hline Buttock width (mm) & 276.07 & 291.18 & $<0.001$ & 292.26 & 307.68 & $<0.001$ & 304.88 & 318.17 & $<0.001$ \\
\hline One-thigh thickness (mm) & 103.77 & 105.71 & 0.064 & 110.57 & 113.89 & 0.001 & 116.91 & 116.28 & 0.521 \\
\hline Two-thigh thickness (mm) & 208.88 & 201.19 & $<0.001$ & 216.91 & 210.75 & $<0.001$ & 224.05 & 216.12 & $<0.001$ \\
\hline Popliteal height (mm) & 371.82 & 362.56 & $<0.001$ & 389.71 & 369.69 & $<0.001$ & 404.71 & 375.55 & $<0.001$ \\
\hline Knee height (mm) & 465.70 & 452.58 & $<0.001$ & 487.95 & 461.04 & $<0.001$ & 505.18 & 467.97 & $<0.001$ \\
\hline Buttock-popliteal length (mm) & 381.77 & 401.25 & $<0.001$ & 404.65 & 414.44 & $<0.001$ & 420.62 & 424.76 & 0.002 \\
\hline Buttock-knee length (mm) & 483.83 & 497.61 & $<0.001$ & 509.46 & 513.36 & 0.017 & 527.07 & 525.32 & 0.242 \\
\hline Sitting height $(\mathrm{mm})$ & 759.22 & 775.88 & $<0.001$ & 793.01 & 802.02 & $<0.001$ & 824.39 & 821.03 & 0.087 \\
\hline Sitting eye height (mm) & 643.93 & 660.97 & $<0.001$ & 677.98 & 685.96 & $<0.001$ & 711.48 & 702.42 & $<0.001$ \\
\hline Sitting elbow height ( $\mathrm{mm})$ & 189.27 & 197.21 & $<0.001$ & 201.15 & 207.82 & $<0.001$ & 210.07 & 216.91 & $<0.001$ \\
\hline
\end{tabular}

(ii) This study showed a significant gender difference in all anthropometric dimensions.

(iii) This study showed a significant difference among different ethnicities in most anthropometric dimensions.

\section{Conflict of Interests}

The authors declare that there is no conflict of interests regarding the publication of this paper.

\section{Acknowledgments}

The authors are grateful to all students who participated in this study and their parents.

\section{References}

[1] B.-S. Liu, "Incorporating anthropometry into design of earrelated products," Applied Ergonomics, vol. 39, no. 1, pp. 115-121, 2008.

[2] C. D. Wickens, J. D. Lee, Y. Liu, and S. E. G. Becker, An Introduction to Human Factors Engineering, Pearson Education, Upper Saddle River, NJ, USA, 2nd edition, 2004.

[3] B. Kayis and A. F. Özok, "The anthropometry of Turkish army men," Applied Ergonomics, vol. 22, no. 1, pp. 49-54, 1991.
[4] B. Y. Jeong and K. S. Park, "Sex differences in anthropometry for school furniture design," Ergonomics, vol. 33, no. 12, pp. 1511$1521,1990$.

[5] Y.-C. Lin, M.-J. J. Wang, and E. M. Wang, "The comparisons of anthropometric characteristics among four peoples in East Asia," Applied Ergonomics, vol. 35, no. 2, pp. 173-178, 2004.

[6] S. A. Oyewole, J. M. Haight, and A. Freivalds, "The ergonomic design of classroom furniture/computer work station for first graders in the elementary school," International Journal of Industrial Ergonomics, vol. 40, no. 4, pp. 437-447, 2010.

[7] N. K. Ahmad Nazif, S. Ezrin Hani, C. K. Lee, and H. Ahmad Rasdan, "A study on the suitability of science laboratory furniture in Malaysian secondary school," in Proceedings of the Asia Pacific Symposium on Advancements in Ergonomics and Safety (ERGOSYM '11), Perlis, Malaysia, December 2011.

[8] H. T. E. Hertzberg, "The conference on standardization of anthropometric techniques and terminology (a report)," American Journal of Physical Anthropology, vol. 28, no. 1, pp. 1-16, 1968.

[9] N. B. Diep, Evaluation of fitness between school furniture and children body size in two primary schools in Haiphong, Vietnam [M.S. thesis], Department of Human Work Science, Division of Industrial Ergonomics, Lulea University of Technology, Luleå, Sweden, 2003.

[10] E. Geldhof, D. De Clercq, I. De Bourdeaudhuij, and G. Cardon, "Classroom postures of 8-12 year old children," Ergonomics, vol. 50, no. 10, pp. 1571-1581, 2007.

[11] S. Milanese and K. Grimmer, "School furniture and the user population: an anthropometric perspective," Ergonomics, vol. 47, no. 4, pp. 416-426, 2004. 
[12] C. Parcells, M. Stommel, and R. P. Hubbard, "Mismatch of classroom furniture and student body dimensions: empirical findings and health implications," Journal of Adolescent Health, vol. 24, no. 4, pp. 265-273, 1999.

[13] L. Saarni, C.-H. Nygård, A. Kaukiainen, and A. Rimpelä, "Are the desks and chairs at school appropriate?" Ergonomics, vol. 50, no. 10, pp. 1561-1570, 2007.

[14] G. Bolstad, B. Benum, and A. Rokne, "Anthropometry of Norwegian light industry and office workers," Applied Ergonomics, vol. 32, no. 3, pp. 239-246, 2001.

[15] M.-J. J. Wang, E. M.-Y. Wang, and Y.-C. Lin, "The anthropometric database for children and young adults in Taiwan," Applied Ergonomics, vol. 33, no. 6, pp. 583-585, 2002.

[16] L. R. Prado-León, R. Avila-Chaurand, and E. L. GonzálezMuñoz, "Anthropometric study of Mexican primary school children," Applied Ergonomics, vol. 32, no. 4, pp. 339-345, 2001.

[17] R. G. Snyder, L. W. Schneider, C. L. Owings, H. M. Reynolds, D. H. Golomb, and M. A. Schork, "Anthropometry of Infants, Students, and Youth to Age 18 for Product Safety Design," Final report 48109, Highway safety research institute, The University of Michigan, Ann Arbor, Mich, USA, 1977.

[18] A. Van Lierop, N. V. Nam, C. Doak et al., "Regional clustering of anthropometric dimensions of primary school children in rural and suburban Vietnam," Asia Pacific Journal of Clinical Nutrition, vol. 17, no. 4, pp. 603-607, 2008.

[19] G. Panagiotopoulou, K. Christoulas, A. Papanckolaou, and K. Mandroukas, "Classroom furniture dimensions and anthropometric measures in primary school," Applied Ergonomics, vol. 35, no. 2, pp. 121-128, 2004.

[20] L. P. A. Steenbekkers and J. F. M. Molenbroek, "Anthropometric data of children for non-specialist users," Ergonomics, vol. 33, no. 4, pp. 421-429, 1990.

[21] G. J. A. Van't Loo, Anthropometric Data for Students in Primary and Secondary Guidance Schools in the Maldives Republic, UNESCO Regional Office for Education, Bangkok, Thailand, 1975.

[22] O. Shrestha, S. Bhattacharya, N. Jha et al., "Cranio facial anthropometric measurements among Rai and Limbu community of Sunsari District, Nepal," Nepal Medical College Journal, vol. 11, no. 3, pp. 183-185, 2009.

[23] S. Hisham, C. R. Mamat, and M. A. Ibrahim, "Multivariate statistical analysis for race variation from foot anthropometry in the Malaysian population," Australian Journal of Forensic Sciences, vol. 44, no. 3, pp. 285-293, 2012.

[24] S. J. Mirmohammadi, R. Hafezi, A. H. Mehrparvar et al., "An epidemiologic study on anthropometric dimensions of 711-year-old Iranian children: considering ethnic differences," Ergonomics, vol. 56, no. 1, pp. 90-102, 2013.

[25] American Anthropological Association, "A Brief History of the OMB Directive 15," 1997.

[26] M. Tunay and K. Melemez, "An analysis of biomechanical and anthropometric parameters on classroom furniture design," African Journal of Biotechnology, vol. 7, no. 8, pp. 1081-1086, 2008.

[27] M. Jahanshahi, M. J. Golalipour, and K. Heidari, "The effect of ethnicity on facial anthropometry in Northern Iran," Singapore Medical Journal, vol. 49, no. 11, pp. 940-943, 2008.

[28] P. V. V. Hamill, T. A. Drizd, and C. L. Johnson, "Physical growth: National Center for Health Statistics percentiles," American Journal of Clinical Nutrition, vol. 32, no. 3, pp. 607-629, 1979.
[29] S. M. Sirajuddin, R. Duggirala, and M. H. Crawford, "Population structure of the Chenchu and other south Indian tribal groups: relationships between genetic, anthropometric, dermatoglyphic, geographic, and linguistic distances," Human Biology, vol. 66, no. 5, pp. 865-884, 1994.

[30] S. A. Smith and B. J. Norris, "Changes in the body size of UK and US children over the past three decades," Ergonomics, vol. 47, no. 11, pp. 1195-1207, 2004.

[31] M. A. Mououdi and A. R. Choobineh, "Static anthropometric characteristics of students age range six-11 in Mazandaran province/Iran and school furniture design based on ergonomics principles," Applied Ergonomics, vol. 28, no. 2, pp. 145-147, 1997.

[32] I. Dianat, M. A. Karimi, A. Asl Hashemi, and S. Bahrampour, "Classroom furniture and anthropometric characteristics of Iranian high school students: proposed dimensions based on anthropometric data," Applied Ergonomics, vol. 44, no. 1, pp.101$108,2013$.

[33] S. J. Mirmohammadi, A. H. Mehrparvar, S. Jafari, and M. Mostaghaci, "An assessment of the anthropometric data of Iranian University Students," International Journal of Occupational Hygiene, vol. 3, no. 2, pp. 85-89, 2011.

[34] M. Mokdad and M. Al-Ansari, "Anthropometrics for the design of Bahraini school furniture," International Journal of Industrial Ergonomics, vol. 39, no. 5, pp. 728-735, 2009.

[35] M. Y. Rosnah, H. M. Rizal, and S. A. R. Sharifah-Norazizan, "Anthropometry dimensions of older Malaysians: comparison of age, gender and ethnicity," Asian Social Science, vol. 5, no. 6, pp. 133-140, 2009. 


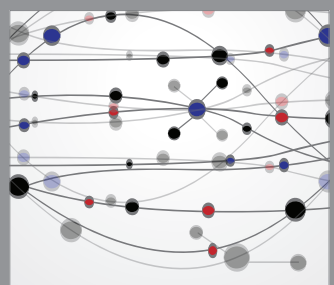

The Scientific World Journal
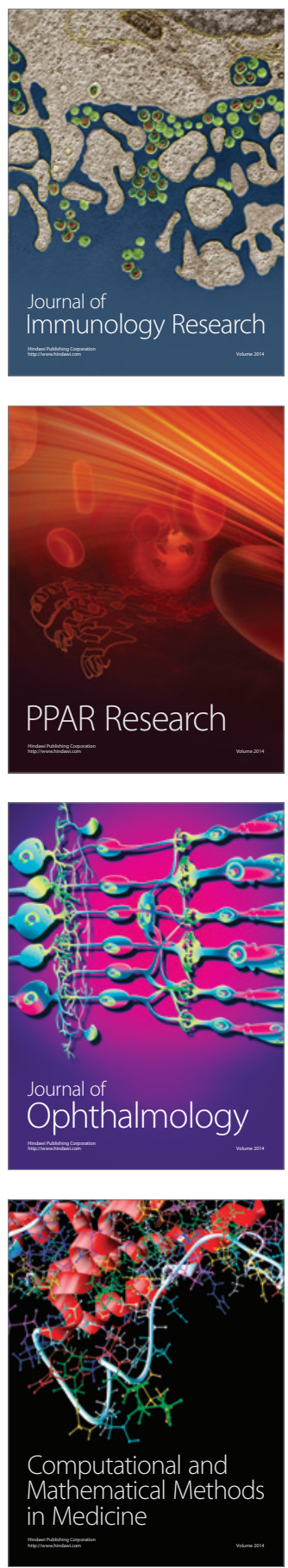

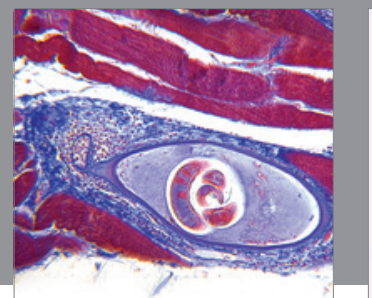

Gastroenterology

Research and Practice
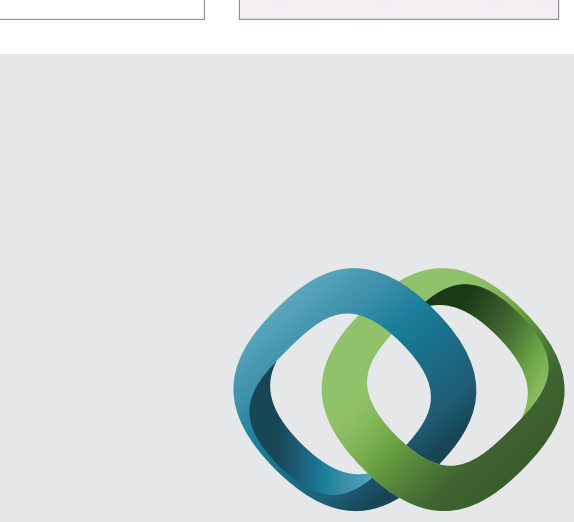

\section{Hindawi}

Submit your manuscripts at

http://www.hindawi.com
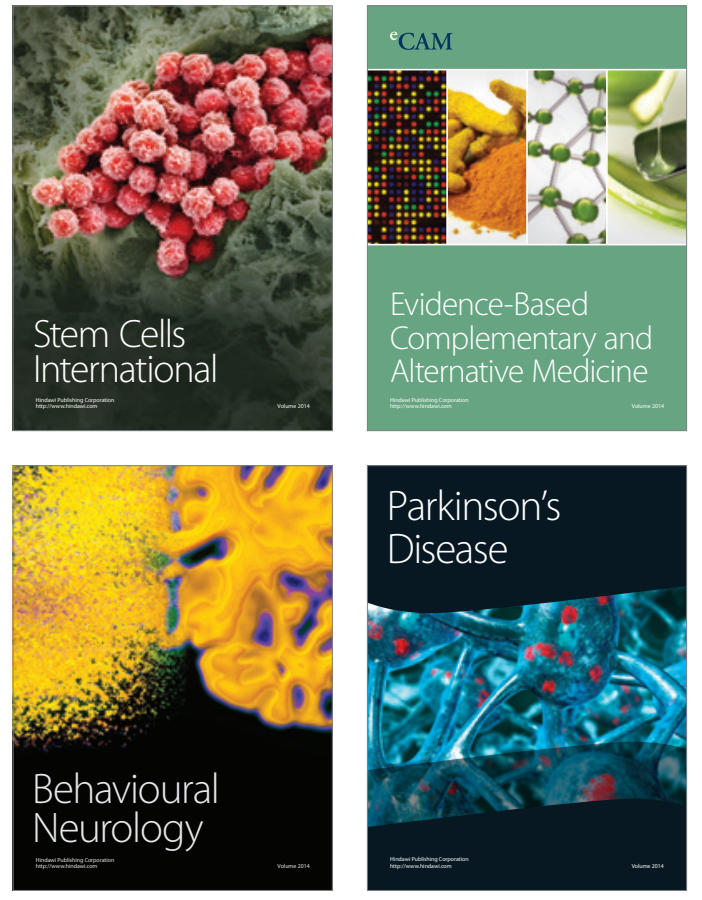
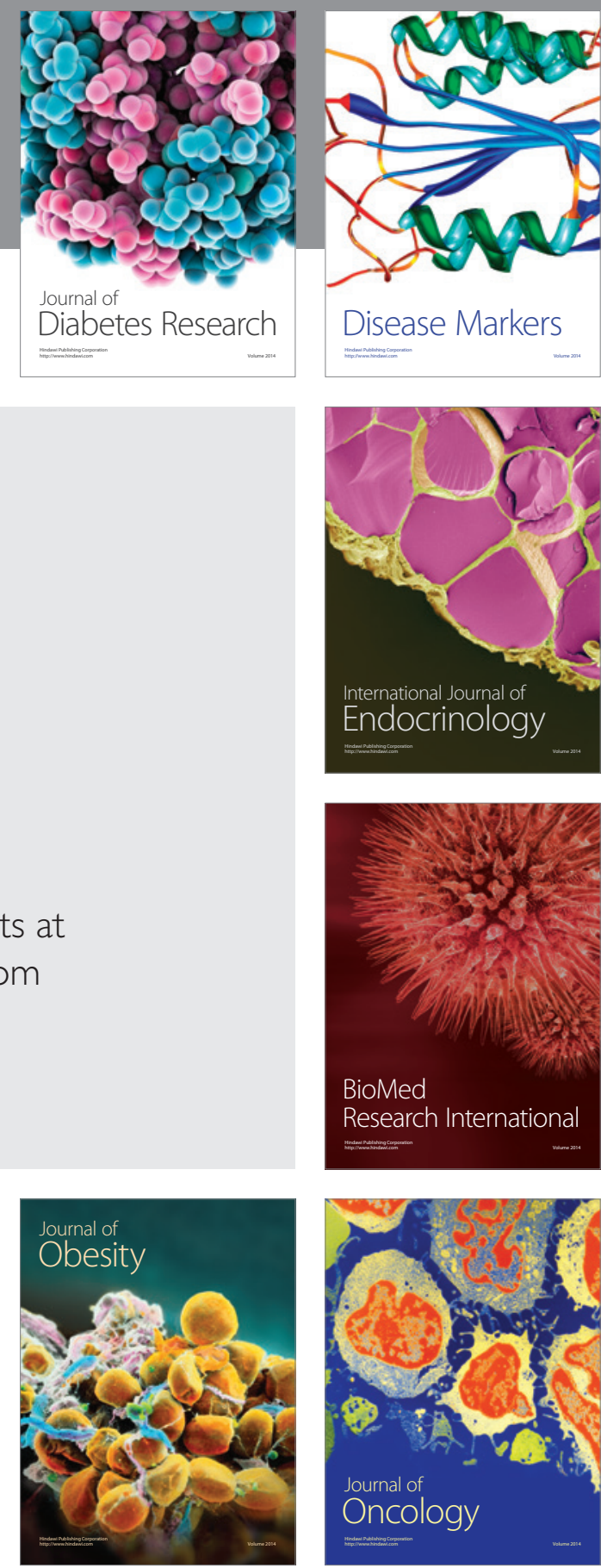

Disease Markers
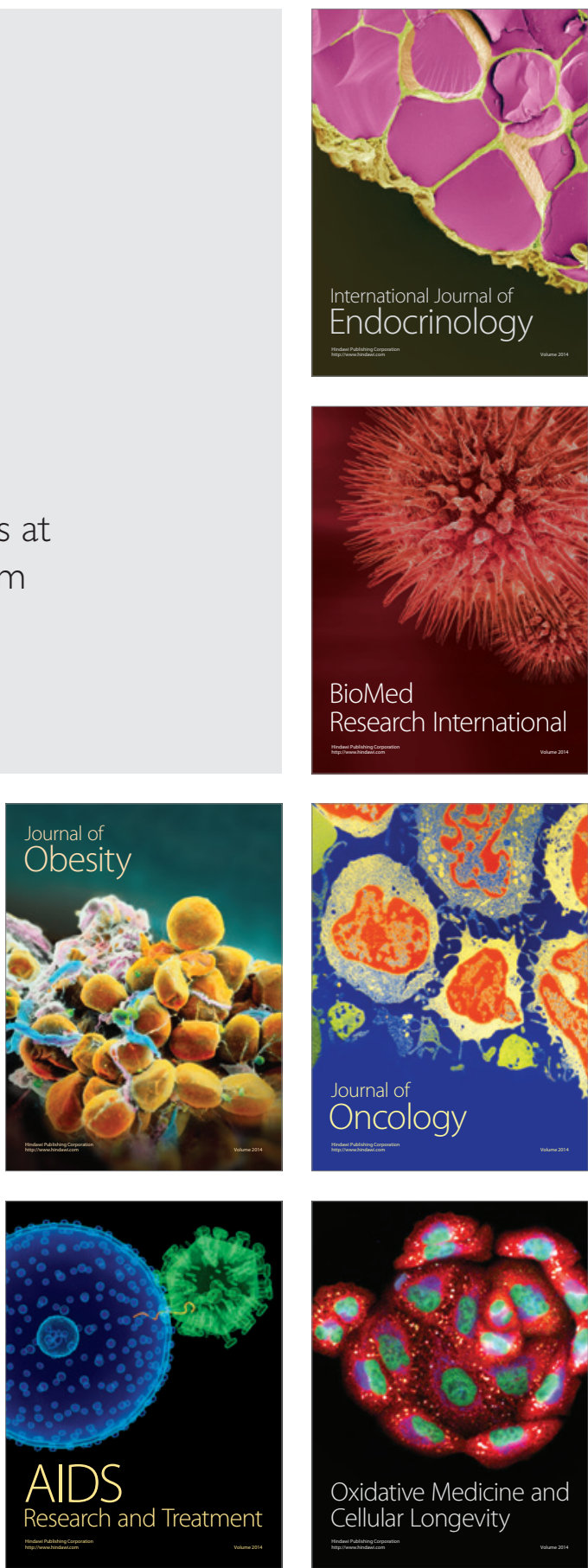